This item was submitted to Loughborough's Research Repository by the author.

Items in Figshare are protected by copyright, with all rights reserved, unless otherwise indicated.

\title{
Null limits of generalised Bonnor-Swaminarayan solutions
}

PLEASE CITE THE PUBLISHED VERSION

LICENCE

CC BY-NC-ND 4.0

\section{REPOSITORY RECORD}

Podolsky, J., and J.B. Griffiths. 2019. "Null Limits of Generalised Bonnor-swaminarayan Solutions". figshare. https://hdl.handle.net/2134/757. 


\title{
Null limits of generalised Bonnor-Swaminarayan solutions
}

\author{
J. Podolský* \\ Institute of Theoretical Physics, Charles University, \\ V Holešovičkách 2, 18000 Prague 8, Czech Republic. \\ and J. B. Griffiths ${ }^{\dagger}$ \\ Department of Mathematical Sciences, Loughborough University \\ Loughborough, Leics. LE11 3TU, U.K.
}

April 27, 2000

\begin{abstract}
The Bonnor-Swaminarayan solutions are boost-rotation symmetric space-times which describe the motion of pairs of accelerating particles which are possibly connected to strings (struts). In an explicit and unified form we present a generalised class of such solutions with a few new observations. We then investigate the possible limits in which the accelerations become unbounded. The resulting space-times represent spherical impulsive gravitational waves with snapping or expanding cosmic strings. We also obtain an exact solution for a snapping string of finite length.
\end{abstract}

Keywords: Accelerating particles, cosmic strings, impulsive spherical wave.

\section{Introduction}

We have recently shown [1] that expanding impulsive spherical gravitational waves may be considered as impulsive limits of the Robinson-Trautman family of vacuum type $\mathrm{N}$ space-times. Such waves may be explicitly constructed using Penrose's method [2] of cutting Minkowski (or de Sitter or anti-de Sitter) space along a null cone and re-attaching the two parts with a suitable warp. However, particular solutions with these properties may also be obtained as limiting cases of certain known solutions which have boost-rotation symmetry. (The first example of this procedure was presented by Bičák and Schmidt at the end of section 5 of [3].) The purpose of the present work is to list some particular boost-rotation symmetric solutions in a suitable form, and to investigate their possible impulsive wave limits in detail.

Boost-rotation symmetric space-times are the only explicitly known exact solutions of Einstein's vacuum field equations which describing moving particles, are radiative and asymptotically flat in the sense that they admit global, though not complete, smooth null infinity, as well as smooth spacelike and timelike infinities. The general properties of such solutions have been extensively investigated and reviewed by Bičák and Schmidt [4] (see also [5] and [6]), and need not be repeated here.

Here, we will concentrate on the Bonnor-Swaminarayan (BS) solutions [7] which are specific boost-rotation symmetric solutions. These are particularly appropriate to consider in the null limit in which impulsive waves will arise. However, some related solutions also have similar limits. We will therefore review the BS and related solutions in a unified way in the next two sections, adapting the notation to our requirements and making a few new observations. We will then investigate their impulsive limits in section 4.

${ }^{*}$ E-mail: Podolsky@mbox.troja.mff.cuni.cz

${ }^{\dagger}$ E-mail: J.B.Griffiths@Lboro.ac.uk 


\section{The Bonnor-Swaminarayan solutions}

The BS solutions [7] can be described by the line element

$$
\mathrm{d} s^{2}=-\mathrm{e}^{\lambda} \mathrm{d} \rho^{2}-\rho^{2} \mathrm{e}^{-\mu} \mathrm{d} \phi^{2}+\left(\zeta^{2}-\tau^{2}\right)^{-1}\left[\mathrm{e}^{\mu}(\zeta \mathrm{d} \tau-\tau \mathrm{d} \zeta)^{2}-\mathrm{e}^{\lambda}(\zeta \mathrm{d} \zeta-\tau \mathrm{d} \tau)^{2}\right],
$$

where

$$
\begin{aligned}
\mu= & -\frac{2 m_{1}}{A_{1} R_{1}}-\frac{2 m_{2}}{A_{2} R_{2}}+4 m_{1} A_{1}+4 m_{2} A_{2}+B \\
\lambda= & 8 m_{1} m_{2} \frac{A_{1}^{3} A_{2}^{3}\left(R_{1}-R_{2}\right)^{2}}{\left(A_{2}^{2}-A_{1}^{2}\right)^{2} R_{1} R_{2}}-\frac{2 m_{1} m_{2}}{A_{1} A_{2} R_{1} R_{2}} \\
& -\left(\frac{m_{1}^{2}}{A_{1}^{2} R_{1}^{4}}+\frac{m_{2}^{2}}{A_{2}^{2} R_{2}^{4}}\right) \rho^{2}\left(\zeta^{2}-\tau^{2}\right)+2\left(\frac{m_{1} A_{1}}{R_{1}}+\frac{m_{2} A_{2}}{R_{2}}\right)\left(\rho^{2}+\zeta^{2}-\tau^{2}\right)+B,
\end{aligned}
$$

in which

$$
R_{i}=\frac{1}{2} \sqrt{\left(\rho^{2}+\zeta^{2}-\tau^{2}-\frac{1}{A_{i}^{2}}\right)^{2}+\frac{4}{A_{i}^{2}} \rho^{2}} \quad(i=1,2) .
$$

For this line element, the metric functions $\mu$ and $\lambda$ depend only on $\rho^{2}$ and $\zeta^{2}-\tau^{2}$. This dependence exhibits explicitly the rotation and boost symmetry.

The detailed physical interpretation of this space-time was described in [7] and [8] (a summary is given in [9]). In general, it represents the motion of two pairs of uniformly accelerating particles which are possibly connected to conical singularities (strings or struts). The particles are located symmetrically, two on the positive and two on the negative $\zeta$-axis. However, the point "masses" are not black holes, but are of the type that are usually described as "Curzon-Chazy" particles. The radiative properties of the BS solutions have been extensively studied by Bičák $[10,11,5,6]$.

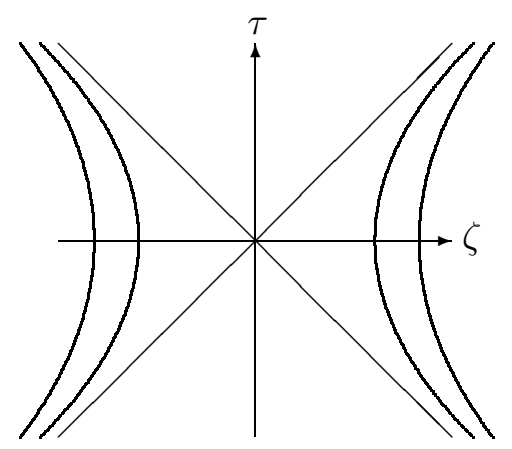

Figure 1: A space-time diagram of the BS solutions

The above solution contains five arbitrary constants $m_{1}, m_{2}, A_{1}, A_{2}$ and $B$. These determine the mass and acceleration of each particle and the singularity structure on the axis of symmetry $\rho=0$. Each pair contains two particles with masses $m_{i}$. These are located on the axis at the points where $R_{i}=0$. They thus have hyperbolic worldlines in the $\tau-\zeta$ plane given by

$$
m_{i}: \quad \rho=0, \quad \zeta= \pm \sqrt{\tau^{2}+\frac{1}{A_{i}^{2}}} .
$$

Each particle is uniformly accelerated with respect to a Minkowski background, the accelerations being $\pm A_{i}$ in the $\pm \zeta$ directions (we shall assume $A_{2}>A_{1}>0$ ). The minimum distance of each particle from $\zeta=0$ is given by $\pm A_{i}^{-1}$.

The metric (1) will generally contain conical singularities, representing strings or struts, located on the axis of symmetry. These will occur unless $\lambda+\mu \rightarrow 0$ as $\rho \rightarrow 0$ (see [4]). In fact, it is possible to choose the constants $m_{i}, A_{i}$ and $B$ such that this regularity condition is satisfied on appropriate sections of the axis.

The following cases are of particular interest: 


\section{Case 1}

It is possible to make the choices

$$
m_{1}=\frac{\left(A_{2}^{2}-A_{1}^{2}\right)^{2}}{4 A_{1}^{3} A_{2}^{2}}, \quad m_{2}=-\frac{\left(A_{2}^{2}-A_{1}^{2}\right)^{2}}{4 A_{1}^{2} A_{2}^{3}}, \quad B=0 .
$$

With this, the axis is regular everywhere except at the locations of the point particles.

Figure 2:

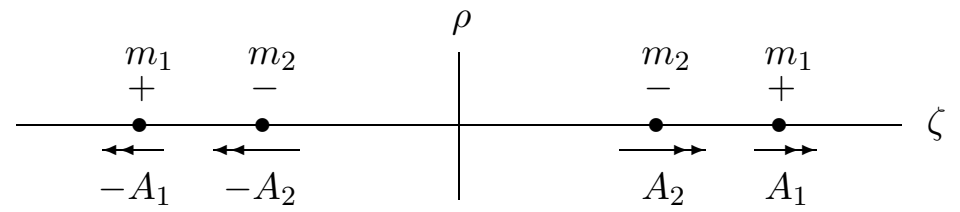

This case had previously been described (in the static region) by Bondi [12] in 1957. For this choice of constants, the outer particles have positive mass, and the inner particles negative mass. The interactions between each pair of particles causes them both to accelerate towards infinity.

\section{Case 2}

The conditions for Case 1 can be relaxed by taking

$$
m_{1}=\frac{\left(A_{2}^{2}-A_{1}^{2}\right)^{2}}{4 A_{1}^{3} A_{2}^{2}}, \quad m_{2} \text { arbitrary, } \quad B=0 .
$$

In this case, the axis is only regular between the particles of each pair and between the two pairs of particles. However, the outer particles are connected to infinity by two semi-infinite strings.

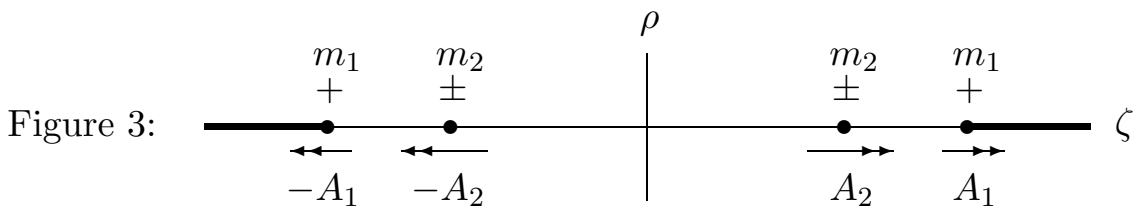

In this case, the outer particles must have positive mass and are "pulled" towards infinity by the tension in the string. However, the inner particles have arbitrary mass which may be positive, negative or zero.

\section{Case 2a}

Of particular interest is the special case of 2 in which $m_{2}=0$. For this choice, the inner particles disappear, and the two remaining particles are pulled towards infinity by semi-infinite strings. This has been described by Bičák, Hoenselaers and Schmidt [13].

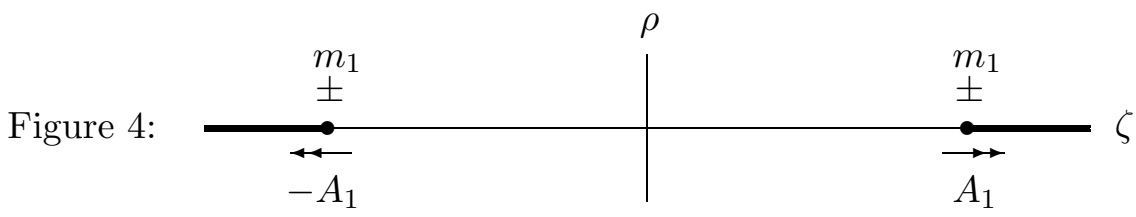

In this particular subcase, however, the restriction that the outer particles must have positive mass no longer occurs. We only require

$$
m_{1} \text { arbitrary, } \quad m_{2}=0, \quad B=0,
$$

so that the remaining particles may also have negative mass, in which case the conical singularity corresponds to a strut rather than a string. (It may be noticed that this subcase can also be obtained in the limit $A_{2} \rightarrow A_{1}$, in which case $m_{1} \rightarrow 0$ and the masses at the end of the conical singularity are replaced by the original inner masses $m_{2}$ which may be arbitrary.) 


\section{Case 3}

A further case can be obtained by imposing the conditions that the axis is regular only between each pair of particles and between each outer particle and infinity. In this case there remains a string between the two inner particles which must have negative mass. However, the outer particles may have arbitrary (positive or negative) mass. The parameters are given by

$$
m_{1} \text { arbitrary, } \quad m_{2}=-\frac{\left(A_{2}^{2}-A_{1}^{2}\right)^{2}}{4 A_{1}^{2} A_{2}^{3}}, \quad B=-4 m_{1} A_{1}-4 m_{2} A_{2} .
$$

Figure 5:

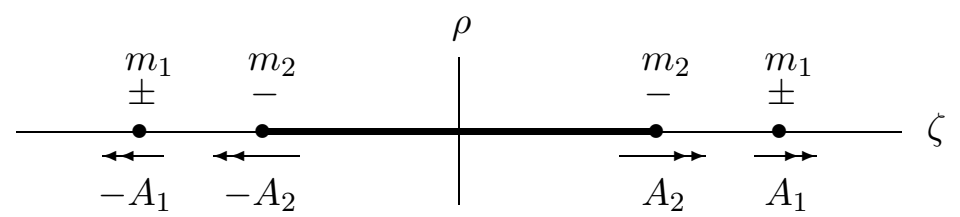

\section{Case 3a}

The special case of 3 arises if $m_{1}=0$. This would result in two particles of negative mass connected by a string. However, the restriction on $m_{2}$ no longer occurs and it may be chosen arbitrarily (as in case 2a above). This has been described in [13]. Alternatively, we may obtain the same situation by taking the limit as $A_{2} \rightarrow A_{1}$ in (8). This subcase is thus given by

$$
m_{1} \text { arbitrary, } \quad m_{2}=0, \quad B=-4 m_{1} A_{1} \text {. }
$$

Figure 6:

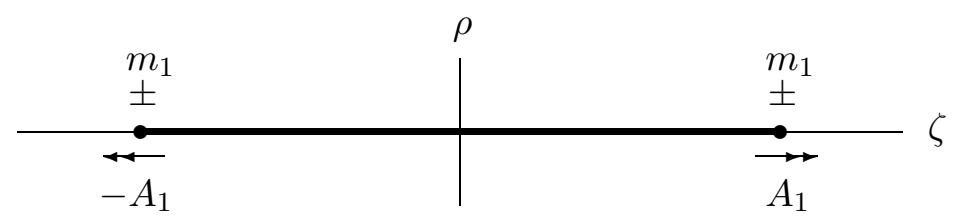

Notice that for the special cases $2 \mathrm{a}$ and $3 \mathrm{a}$ describing only two accelerated particles, the metric functions (2) can be written in a simple form (see [16])

$$
\begin{aligned}
\mu & =\mu_{0}=-\frac{2 m}{A R}+4 m A+B, \\
\lambda & =\lambda_{0}=-\frac{m^{2}}{A^{2} R^{4}} \rho^{2}\left(\zeta^{2}-\tau^{2}\right)+\frac{2 m A}{R}\left(\rho^{2}+\zeta^{2}-\tau^{2}\right)+B,
\end{aligned}
$$

where $B=0$ for case $2 \mathrm{a}$ or $B=-4 m A$ for case $3 \mathrm{a}$,

$$
R=\frac{1}{2} \sqrt{\left(\rho^{2}+\zeta^{2}-\tau^{2}-\frac{1}{A^{2}}\right)^{2}+\frac{4}{A^{2}} \rho^{2}} .
$$

In these expressions, the unnecessary indices have been omitted (i.e. $m=m_{1}, A=A_{1}, R=R_{1}$ ).

\section{Case 4}

A further case has been described in detail in [14] for which the axis is regular everywhere except between the two pairs of particles. The parameters are here given by

$$
m_{1} \text { arbitrary, } \quad m_{2}=-\frac{A_{1}}{A_{2}} m_{1}, \quad B=0 .
$$

In this case the masses of the two particles in each pair must be of opposite sign. If the outer particle has positive (negative) mass, the singularity corresponds to a strut (string).

Figure 7:

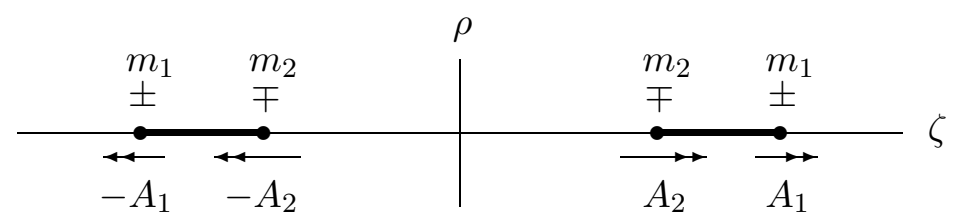




\section{The Bičák-Hoenselaers-Schmidt solutions}

Let us now consider some further solutions that can be obtained from those above by taking suitable limits. A further solution will also be included which contains the special cases above.

\section{Case 2b}

One special limit (described in detail by Bičák, Hoenselaers and Schmidt [13]) of case 2 above that is of particular interest is that in which $A_{1} \rightarrow 0$. In this case, the outer particles, and the strings attached to them, are scaled out to infinity. The significance of this solution is that the remaining particles move freely and are not connected to strings. However, they move under the action of an exterior gravitational field for which the source is at infinity. It may be noted that although $m_{1} \sim A_{1}^{-3}$ in this limit, $R_{1} \sim A_{1}^{-2}$ and $\mu$ is bounded. The resulting solution is given by

$$
\begin{aligned}
& \mu=-\frac{2 m_{2}}{A_{2} R_{2}}+4 m_{2} A_{2}+A_{2}^{2}\left(\rho^{2}-\zeta^{2}+\tau^{2}\right) \\
& \lambda=-\left(\frac{m_{2}^{2}}{A_{2}^{2} R_{2}^{4}}+A_{2}^{4}\right) \rho^{2}\left(\zeta^{2}-\tau^{2}\right)+A_{2}^{2}\left(\rho^{2}+\zeta^{2}-\tau^{2}\right)+\frac{2 m_{2}}{A_{2} R_{2}}\left(2 A_{2}^{2} \rho^{2}+1\right)-4 m_{2} A_{2} .
\end{aligned}
$$

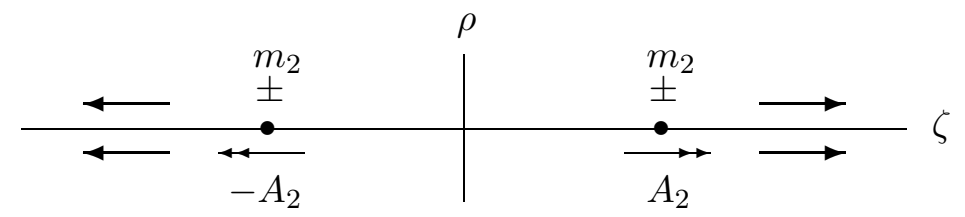

\section{Case 4a}

We can also consider the limit of case 4 in which the $A_{2} \rightarrow A_{1}$ so that the two particles in each pair approach each other. This procedure has been described by Bičák, Hoenselaers and Schmidt [14]. It is convenient to introduce the parameter $M_{01}=2\left(\frac{m_{1}}{A_{1}}+\frac{m_{2}}{A_{2}}\right)$ which, with the constraint (12), becomes $M_{01}=\frac{2 m_{1}}{A_{1} A_{2}^{2}}\left(A_{2}^{2}-A_{1}^{2}\right)$. In this limit, Minkowski space-time is obtained unless the parameter $m_{1}$ is rescaled in such a way that $M_{01}$ is kept constant. This particular solution is given by

$$
\begin{aligned}
\mu= & -\frac{M_{01}}{R}+\frac{M_{01}}{4 A^{2} R^{3}}\left(\rho^{2}-\zeta^{2}+\tau^{2}+\frac{1}{A^{2}}\right) \\
\lambda=- & -\frac{M_{01}^{2}}{64 R^{8}} \rho^{2}\left(\zeta^{2}-\tau^{2}\right)\left[\left(\left(\rho^{2}+\zeta^{2}-\tau^{2}\right)^{2}-\frac{1}{A^{4}}\right)^{2}-\frac{2}{A^{4}} \rho^{2}\left(\zeta^{2}-\tau^{2}\right)\right] \\
& \quad-\frac{M_{01}}{4 R^{3}}\left(\rho^{2}+\zeta^{2}-\tau^{2}\right)\left(\rho^{2}-\zeta^{2}+\tau^{2}+\frac{1}{A^{2}}\right)
\end{aligned}
$$

It may be noticed that an analogous limit of case 1 does not exist as there is no freedom to rescale the mass $m_{1}$ (see (5)) and Minkowski space is obtained as the two particles coalesce.

Figure 9:

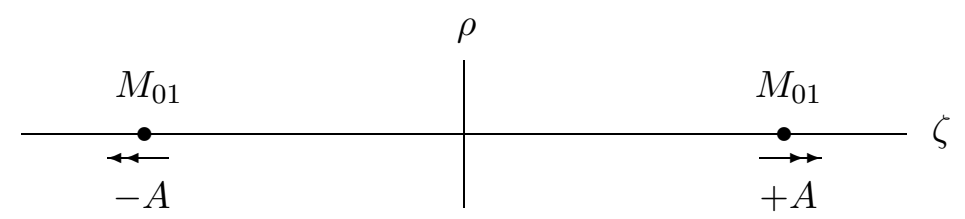

As shown in [14], the metric describes a combination of monopole and dipole terms, so that the resulting particle has been referred to as a Curzon-Chazy (01)-pole particle (for this reason we have denoted the parameter by $M_{01}$ ). The space-time simply contains two such particles. The situation looks similar to that for case $2 \mathrm{~b}$ above. However, in this case there is no external fields and the particles are accelerated by their internal dipole component. 


\section{Multipole particles}

In [14] other boost-rotationally symmetric spacetimes generalising the Bonnor-Swaminarayan solution (1-2) were found. These represent the fields of two accelerating particles with arbitrary multipole structure attached to conical singularities as in the cases $2 \mathrm{a}$ and $3 \mathrm{a}$ above. Moreover, for a special choice of parameters the space-times may be free of conical singularities as in the case $4 \mathrm{a}$, or $2 \mathrm{~b}$ but without an external field.

This class of solutions was presented in [14] using prolate spheroidal coordinates related to the Weyl form of the boost-rotational symmetric metric. We will give here an explicit closed form of these solutions in terms of the metric functions for the line element (1). However, the expression for $\lambda$ is presented here in a much simpler form than that given in [14]. The derivation of this new expression, and the relation between the two forms, are contained in the appendix. In addition, using the scaling property of the Weyl metric, we can include the acceleration parameter $A$ explicitly (this parameter does not appear in [14], in which the scaling was used to put $A=\frac{1}{2}$ ). In this new form, the general class of multipole solutions is given by

$$
\begin{aligned}
& \mu= 2 \sum_{n=0}^{\infty} M_{n} \frac{P_{n}}{(x-y)^{n+1}}+C, \\
& \lambda=-2 \sum_{k, l=0}^{\infty} M_{k} M_{l} \frac{(k+1)(l+1)}{(k+l+2)} \frac{\left(P_{k} P_{l}-P_{k+1} P_{l+1}\right)}{(x-y)^{k+l+2}} \\
& \quad-\left(\frac{x+y}{x-y}\right) \sum_{n=0}^{\infty} \frac{M_{n}}{2^{n}} \sum_{l=0}^{n}\left(\frac{2}{x-y}\right)^{l} P_{l}+D,
\end{aligned}
$$

where the constants $M_{n}$ represent the multipole moments, the argument of the Legendre polynomials $P_{n}$ is $\alpha=(1-x y) /(x-y)$, and $C, D$ are constants to be specified below. For the prolate spheroidal coordinates $x$ and $y$ and for $\alpha$ in (15) one has to substitute from the relations

$$
\begin{aligned}
& x-y=4 A^{2} R, \quad x+y=2 A^{2}\left(\rho^{2}+\zeta^{2}-\tau^{2}\right), \\
& \alpha=\frac{1}{2 R}\left(\rho^{2}-\zeta^{2}+\tau^{2}+\frac{1}{A^{2}}\right),
\end{aligned}
$$

where $R$ is given by (11). These imply the useful formulae

$$
\left(x^{2}-1\right)\left(1-y^{2}\right)=16 A^{4} \rho^{2}\left(\zeta^{2}-\tau^{2}\right), \quad 1+x y=2 A^{2}\left(\zeta^{2}-\tau^{2}-\rho^{2}\right) .
$$

It now remains to set the values of the constants $C$ and $D$. To ensure that the space-time is regular on the "roof" $\zeta^{2}-\tau^{2}=0$, it is necessary that $\mu=\lambda$ at $\rho=0=\zeta^{2}-\tau^{2}$ (see [4]). This yields explicitly that $D=C+\sum_{n=0}^{\infty} M_{n} / 2^{n}$. Moreover, the metric is regular on the axis provided $\mu+\lambda=0$ at $\rho=0$. Regularity of the axis between the two particles, analogous to the case 2 a (see Fig. 4), requires that

$$
C=-\sum_{n=0}^{\infty} \frac{M_{n}}{2^{n}}, \quad D=0 .
$$

In this case there is generally a string connecting the particles to infinity. The alternative situation, analogous to case 3a (see Fig. 6), in which there is a string between the particles and the axis is regular outside is given by

$$
C=0, \quad D=\sum_{n=0}^{\infty} \frac{M_{n}}{2^{n}} .
$$

The axis is obviously regular everywhere, except at the particle, if the combination of multipole moments satisfies the condition that $\sum_{n=0}^{\infty} M_{n} / 2^{n}=0$ so that $C=0=D$. This was pointed out in [14]. 
Considering only the case $n=0$ in (15), we recover the previous formula (10) for the monopole BS particles as in case 2a or 3a with identification $M_{0}=-4 m A$.

It is also straightforward to write an explicit solution representing accelerated dipole particles with the single moment $M_{1}$ :

$$
\begin{aligned}
\mu_{1}= & \frac{M_{1}}{16 A^{4} R^{3}}\left(\rho^{2}-\zeta^{2}+\tau^{2}+\frac{1}{A^{2}}\right)+C \\
\lambda_{1}= & \frac{M_{1}^{2}}{512 A^{8} R^{4}}\left(9 \alpha^{4}-10 \alpha^{2}+1\right) \\
& \quad-\frac{M_{1}}{4 R}\left[1+\frac{1}{4 A^{2} R^{2}}\left(\rho^{2}-\zeta^{2}+\tau^{2}+\frac{1}{A^{2}}\right)\right]\left(\rho^{2}+\zeta^{2}-\tau^{2}\right)+D,
\end{aligned}
$$

With these two observations, it may also be seen that case 4a given by (14) is a special case of the general class of solutions (15). It is a combination of both monopole and dipole terms with the identification $2 M_{0}=-M_{1}=-4 A^{2} M_{01}$, so that the constraint $M_{0}+M_{1} / 2=0$ which guarantees the regularity of the axis is automatically satisfied. This is consistent with the interpretation of the solution (14) given in [14]. Indeed, the metric functions $\mu$ and $\lambda$ given by (14) can be written as $\mu=\mu_{0}+\mu_{1}$ and $\lambda=\lambda_{0}+\lambda_{1}+\lambda_{01}$, where $\mu_{0}, \lambda_{0}$ are given by (10), $\mu_{1}, \lambda_{1}$ are given by (19), and $\lambda_{01}=M_{01}^{2} \alpha\left(1-\alpha^{2}\right) / 4 A^{2} R^{3}$.

\section{Limiting cases of the above metrics}

\section{Cases $3 a$ and $2 a$}

A particular limit of case 3 a above in which $m_{2}=0$ and $A_{1} \rightarrow \infty$ was investigated by Bičák and Schmidt [3] in 1989. In this limit, there are only two particles of vanishing mass and their accelerations become infinite. In addition, it is necessary that the parameter $m=m_{1}$ is scaled to zero in such a way that the "monopole moment" $M_{0}=-4 m A$ remains constant. The resulting space-time is flat everywhere except on an expanding sphere and on the strut between the particles. It therefore describes an expanding spherical impulsive gravitational wave that is generated by the two particles which move apart at the speed of light in a Minkowski background and are connected to each other by an expanding strut.

An analogous situation in which (for $\tau>0$ ) two null particles recede from the origin with the speed of light and are connected to infinity by semi-infinite strings was constructed explicitly by Gleiser and Pullin [15] in 1989. This describes the space-time representing an expanding impulsive spherical gravitational wave generated by a snapping cosmic string. Such a solution can also be obtained as a limit of case 2 a above [16]. Again, it is necessary that $m$ is scaled to zero such that $M_{0}$ remains constant. However, as pointed out by Bičák [16], the complete solution rather describes two semi-infinite strings approaching at the speed of light and separating again at the instant at which they collide.

In the remainder of this section, we will investigate similar null limits of all the remaining cases described in sections 2 and 3.

\section{Multipole particles}

We may first consider the null limit of the general class of solutions (15-16). These represent two accelerated particles with arbitrary multipole structure possibly with strings between the particles or with strings connecting each particle to infinity. Here, we let $A \rightarrow \infty$ while all the multipole moments $M_{n}$ are kept constant. In this limit we obtain

$$
\begin{array}{ll}
2 R \rightarrow\left|\rho^{2}+\zeta^{2}-\tau^{2}\right|, & \alpha \rightarrow \frac{\rho^{2}-\zeta^{2}+\tau^{2}}{\left|\rho^{2}+\zeta^{2}-\tau^{2}\right|}, \\
x-y \rightarrow 2 A^{2}\left|\rho^{2}+\zeta^{2}-\tau^{2}\right|, & x+y \rightarrow 2 A^{2}\left(\rho^{2}+\zeta^{2}-\tau^{2}\right), \\
\frac{x+y}{x-y} \rightarrow \operatorname{sign}\left(\rho^{2}+\zeta^{2}-\tau^{2}\right) . &
\end{array}
$$


In this limit, $\mu$ in (15) approaches the constant $C$. Also the first term for $\lambda$ vanishes, and only the contribution $l=0$ in the second term remains finite. Consequently, the metric functions for the solutions which we obtain in the above null limit can be written as

$$
\begin{aligned}
\mu & =C, \\
\lambda & =D-\operatorname{sign}\left(\rho^{2}+\zeta^{2}-\tau^{2}\right) \sum_{n=0}^{\infty} \frac{M_{n}}{2^{n}} .
\end{aligned}
$$

For the particular values of the constants $C$ and $D$ given by (17), this solution appears to be a generalisation of the null limit of case $2 \mathrm{a}$ which describes a situation of a snapping cosmic string. The ends of two semi-infinite cosmic string move in opposite directions with the speed of light, generating an impulsive spherical gravitational wave. However, in this limit, the multipole structure of the initial particles disappears and the solution is characterised by the single constant $M=\sum_{n=0}^{\infty} M_{n} / 2^{n}$. Thus, the null limit for any accelerating particle with a multipole structure is identical to that for a particle with just a monopole term $M_{0}=M$.

Considering the alternative values of the constants given by (18), we obtain a null limit in which a spherical impulsive wave is generated by a finite string whose length is expanding at the speed of light. However, at the ends of the strings the arbitrary multipole structure of the initial "particles" described by the multipole moments $M_{n}$ again disappears. This null limit is thus identical to that of the case $3 \mathrm{a}$ above as originally obtained in [3].

Performing the well-known transformation (see e.g. [4])

$$
\rho=\frac{1}{2}(v-u), \quad \tau= \pm \frac{1}{2}(v+u) \cosh \chi, \quad \zeta=\frac{1}{2}(v+u) \sinh \chi,
$$

we can put the solution (21) into the standard form of boost-rotational symmetric metrics with null coordinates $u$ and $v$,

$$
\mathrm{d} s^{2}=\mathrm{e}^{\lambda} \mathrm{d} u \mathrm{~d} v-\frac{1}{4}(v-u)^{2} \mathrm{e}^{-\mu} \mathrm{d} \phi^{2}-\frac{1}{4}(v+u)^{2} \mathrm{e}^{\mu} \mathrm{d} \chi^{2} .
$$

Since $\rho^{2}+\zeta^{2}-\tau^{2}=-u v$, the metric functions take the following form:

$$
\begin{array}{lll}
\text { snapping string : } & \mu=-\sum_{n=0}^{\infty} \frac{M_{n}}{2^{n}}, & \lambda=[\Theta(u v)-\Theta(-u v)] \sum_{n=0}^{\infty} \frac{M_{n}}{2^{n}}, \\
\text { expanding string : } & \mu=0, & \lambda=2 \Theta(u v) \sum_{n=0}^{\infty} \frac{M_{n}}{2^{n}},
\end{array}
$$

where $\Theta$ is the Heaviside step function. Notice that in both cases $\mu$ is a constant, but there is a discontinuity in the otherwise constant value of $\lambda$ with the step $2 \sum_{n=0}^{\infty} M_{n} / 2^{n}$ on the null cone $u v=0$. However, it is possible to find a transformation to coordinates in which the metric is continuous everywhere. In the region where the functions $\mu$ and $\lambda$ are constant, the transformation

$$
\mathcal{U}=\frac{1}{2} u \mathrm{e}^{\lambda+\mu / 2}, \quad \mathcal{V}=\frac{1}{2} v \mathrm{e}^{-\mu / 2}, \quad \psi=\chi \mathrm{e}^{\mu},
$$

brings the line element (23) into the form

$$
\mathrm{d} s^{2}=4 \mathrm{~d} \mathcal{U} \mathrm{d} \mathcal{V}-\mathcal{A}^{2} \mathrm{~d} \phi^{2}-\mathcal{B}^{2} \mathrm{~d} \psi^{2},
$$

where

$$
\mathcal{A}=\mathcal{V}-\mathcal{U} \mathrm{e}^{-(\lambda+\mu)}, \quad \mathcal{B}=\mathcal{V}+\mathcal{U} \mathrm{e}^{-(\lambda+\mu)} .
$$

This metric is explicitly continuous, including on the null cone given by $\mathcal{U}=0$, even if there has been a discontinuity in $\lambda$ at $u v=0$ in the original coordinates. Such a step is removed by the compensating discontinuity in the expression for $\mathcal{U}$ in (24). 
The solution for a snapping string can thus be written in the continuous form (25). By substituting the corresponding $\lambda$ and $\mu$ into (26), and restricting to the region $v>0$, we obtain

$$
\begin{aligned}
\mathcal{A} & =\mathcal{V}-\left[\Theta(\mathcal{U})+\Theta(-\mathcal{U}) \exp \left(2 \sum_{n=0}^{\infty} \frac{M_{n}}{2^{n}}\right)\right] \mathcal{U} \\
\mathcal{B} & =\mathcal{V}+\left[\Theta(\mathcal{U})+\Theta(-\mathcal{U}) \exp \left(2 \sum_{n=0}^{\infty} \frac{M_{n}}{2^{n}}\right)\right] \mathcal{U} .
\end{aligned}
$$

The metric (25), (27) is exactly of the type constructed previously by Gleiser and Pullin [15] by a different method. It represents an impulsive spherical gravitational wave propagating in the Minkowski universe. However, outside the wave (where $\mathcal{U}<0$ ), there extends the "snapped" cosmic string which is characterized by a deficit angle $(1-\beta) 2 \pi$, where here $\beta=\exp \left(\sum_{n=0}^{\infty} M_{n} / 2^{n}\right)$. This generalizes the previous result presented in [16] which was obtained as the null limit of case 2a describing two accelerated monopole particles. And, as in this particular case, the complete solution has to be extended symmetrically to negative times, as has been argued in [16].

Similarly, we can write the solution for an expanding string in the form (25):

$$
\begin{aligned}
\mathcal{A} & =\mathcal{V}-\left[\Theta(\mathcal{U}) \exp \left(-2 \sum_{n=0}^{\infty} \frac{M_{n}}{2^{n}}\right)+\Theta(-\mathcal{U})\right] \mathcal{U} \\
\mathcal{B} & =\mathcal{V}+\left[\Theta(\mathcal{U}) \exp \left(-2 \sum_{n=0}^{\infty} \frac{M_{n}}{2^{n}}\right)+\Theta(-\mathcal{U})\right] \mathcal{U}
\end{aligned}
$$

In this case there is a string with the deficit angle $\left(1-\beta^{-1}\right) 2 \pi$ in the Minkowski space inside the impulsive wave in the region $\mathcal{U}>0$.

There exists an alternative continuous form of the above solutions. By applying the transformation (24) followed by $U=-2 \mathcal{U}, V=\mathcal{V}, Z=\frac{1}{\sqrt{2}}(\psi+\mathrm{i} \phi)$, we may convert the metric (23) with constant $\lambda$ and $\mu$, in the region $u<0, v>0$, into the form

$$
\mathrm{d} s^{2}=-2|V \mathrm{~d} Z+U H \mathrm{~d} \bar{Z}|^{2}-2 \mathrm{~d} U \mathrm{~d} V,
$$

where $H=-\frac{1}{2} \mathrm{e}^{-(\lambda+\mu)}$ and $U>0$. Performing now a different transformation

$$
\begin{aligned}
U & =-\frac{u v}{u+v} \exp \left[\frac{\lambda}{2}-\chi e^{(\mu-\lambda) / 2}\right], \\
V & =\frac{1}{2}(u+v) \exp \left[\frac{\lambda}{2}+\chi e^{(\mu-\lambda) / 2}\right], \\
Z & =\frac{1}{\sqrt{2}} \frac{v-u}{v+u} \exp \left[-\chi e^{(\mu-\lambda) / 2}+\mathrm{i} \phi e^{-(\mu+\lambda) / 2}\right],
\end{aligned}
$$

of the metric (23) in the region $u>0, v>0$, where $U<0$, we obtain

$$
\mathrm{d} s^{2}=-2 V^{2} \mathrm{~d} Z \mathrm{~d} \bar{Z}-2 \mathrm{~d} U \mathrm{~d} V .
$$

It is now obvious that we can match the two metrics (29) and (31) across the null cone $u=0$, which corresponds to $U=0$, so that the resulting metric

$$
\mathrm{d} s^{2}=-2|V \mathrm{~d} Z+U \Theta(U) H \mathrm{~d} \bar{Z}|^{2}-2 \mathrm{~d} U \mathrm{~d} V
$$

is continuous. This metric is contained within a general class of metrics which describe impulsive spherical gravitational waves (see [2], [17], [18] and [1]). In the general case, the function $H(Z)$ can be obtained as the Schwarzian derivative of a "warp" function $h(Z)$ which permits a geometrical interpretation [19]. The above solution for a snapping string is given by $h=\mathrm{e}^{\beta z}$, where $\beta=\sqrt{2} \exp \left(\sum_{n=0}^{\infty} M_{n} / 2^{n}\right)$ corresponding to $H=-\frac{1}{2} \exp \left(2 \sum_{n=0}^{\infty} M_{n} / 2^{n}\right)$. The alternative case for an expanding string is given by $\beta \rightarrow \beta^{-1}$ corresponding to $H=-\frac{1}{2} \exp \left(-2 \sum_{n=0}^{\infty} M_{n} / 2^{n}\right)$. 


\section{An alternative limit and Case $4 a$}

It can immediately be observed that if the condition $\sum_{n=0}^{\infty} M_{n} / 2^{n}=0$ is satisfied (i.e. when the original metric is regular everywhere on the axis except at the locations of the particles), the corresponding null limit results in a trivial Minkowski spacetime without an impulsive wave. However, it is also possible in this case to perform a more involved null limit which results in a different class of spacetimes than those described by (21). The sum in the second term for $\lambda$ in (15) can be written as

$$
\sum_{n=0}^{\infty} \frac{M_{n}}{2^{n}} \sum_{l=0}^{n}\left(\frac{2}{x-y}\right)^{l} P_{l}=\sum_{n=0}^{\infty} \frac{M_{n}}{2^{n}}+\left(\frac{2 \alpha}{x-y}\right) \sum_{n=1}^{\infty} \frac{M_{n}}{2^{n}}+\sum_{l=2}^{n}\left(\frac{2}{x-y}\right)^{l} P_{l} \sum_{n=l}^{\infty} \frac{M_{n}}{2^{n}} .
$$

When $\sum_{n=0}^{\infty} M_{n} / 2^{n}=0$, the second term in the expansion (33)

$$
\left(\frac{2 \alpha}{x-y}\right) \sum_{n=1}^{\infty} \frac{M_{n}}{2^{n}}=-\frac{2 M_{0} \alpha}{x-y}
$$

becomes dominant, and a different null limit can be obtained. Considering that $x-y \sim A^{2}$ in the null limit $A \rightarrow \infty$, we may here rescale the remaining monopole moment $M_{0}$ in such a way that $M=M_{0} A^{-2}$ remains constant. With these assumptions and using (20) we can write the final form of the non-trivial null limit as

$$
\begin{aligned}
\mu & =\frac{M}{\left|\rho^{2}+\zeta^{2}-\tau^{2}\right|}, \\
\lambda & =-M^{2} \frac{\rho^{2}\left(\zeta^{2}-\tau^{2}\right)}{\left(\rho^{2}+\zeta^{2}-\tau^{2}\right)^{4}}+M \frac{\rho^{2}-\zeta^{2}+\tau^{2}}{\left(\rho^{2}+\zeta^{2}-\tau^{2}\right)^{2}} \operatorname{sign}\left(\rho^{2}+\zeta^{2}-\tau^{2}\right) .
\end{aligned}
$$

This represents a unique alternative solution which can be obtained in the null limit described above if $\sum_{n=0}^{\infty} M_{n} / 2^{n}=0$, after a suitable rescaling of the multipole moments. However, even in this more involved limit, the multipole structure is again completely "erased". Moreover, the metric is not acceptable as a description of an impulsive gravitational wave since it is singular on the null cone $\rho^{2}+\zeta^{2}=\tau^{2}$. It may further be noted that when $M_{0}=0$, the solution is simply Minkowski space.

Notice finally, that the solution (35) exactly corresponds to that which is obtained in the null limit $A \rightarrow \infty$ from the metric (14) of case 4 a with the identification $M=-2 M_{01}$.

\section{Cases 2b, 1, 2 and 3}

Concerning case $2 \mathrm{~b}$, it may immediately be seen from (13) that the null limit in which $A_{2} \rightarrow \infty$ does not exist. The terms in the metric functions which represent the external field responsible for the acceleration diverge.

We have now considered all the above special cases which involve just two accelerating particles. We therefore return to the remaining cases which involve two pairs of distinct particles. In all these cases, it is only possible to consider null limits as $A_{2} \rightarrow \infty$ (since $A_{2}>A_{1}$ ). In cases 1 and 2 , this limit diverges $\left(\mu \sim A_{2}^{2}\right)$. However, in case 3 , the limit for $\mu$ is given by

$$
\mu=-\frac{2 m_{1}}{A_{1} R_{1}}-\frac{1}{A_{1}^{2}\left|\rho^{2}+\zeta^{2}-\tau^{2}\right|},
$$

and this can be seen to diverge on the expanding spherical surface $\rho^{2}+\zeta^{2}=\tau^{2}$. Null limits of the cases 1, 2 and 3 must therefore be considered to be unphysical. 


\section{Case 4}

It now only remains to consider the limit of case 4 . We again consider the limit as $A_{2} \rightarrow \infty$, but we can now do this while scaling $m_{2}$ to zero in such a way that $A_{2} m_{2}=-A_{1} m_{1}=$ constant. In this case the limit is given by

$$
\begin{aligned}
\mu & =-\frac{2 m}{A R}, \\
\lambda & =-\frac{m^{2}}{A^{2} R^{4}} \rho^{2}\left(\zeta^{2}-\tau^{2}\right)+\frac{2 m A}{R}\left(\rho^{2}+\zeta^{2}-\tau^{2}\right)-\operatorname{sign}\left(\rho^{2}+\zeta^{2}-\tau^{2}\right) 4 m A,
\end{aligned}
$$

in which $R=R_{1}$ is given by (11), and the subscript 1 has been omitted from $m_{1}, A_{1}$ and $R_{1}$. This solution contains two free parameters $m$ and $A$ (although it is not possible to consider the limit as $A \rightarrow 0$ while keeping $m$ finite). It represents a snapping string of finite length whose outer ends are accelerating apart as shown in figure 10.

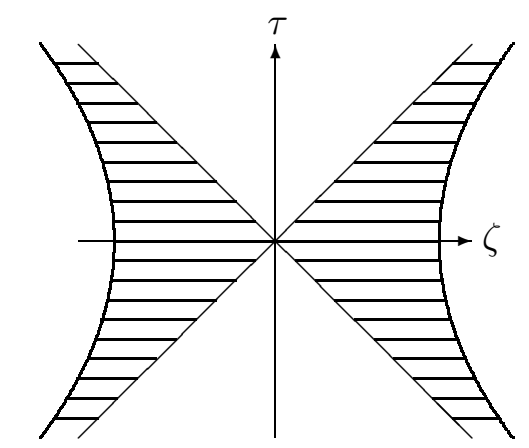

Figure 10. A space-time diagram for a finite snapping string

The two outer particles of mass $m$ are caused to accelerate by the strut or string (there is a deficit angle if $m<0$ ) connecting them. However, in this case, the finite strut breaks (or the string snaps) at its midpoint and the two broken ends separate at the speed of light.

Apart from the step change in $\lambda$, the metric (36) is identical to that of cases 2a and 3a as given in (10). For the general family of metrics (1), the curvature tensor components are linear in $\lambda$, and its discontinuities give rise to impulsive components on the null cone. In fact, the Ricci tensor components still vanish everywhere. However, an impulsive component arises in the Weyl tensor on the null cone ${ }^{1}$. This is interpreted as describing an expanding spherical impulsive gravitational wave that is generated by the snapping of the string.

The complete solution of course is time symmetric as illustrated in figure 10. This situation resembles that of the known solutions for snapping infinite cosmic strings [15], [16]. However, this solution differs in that it is not flat on both sides of the impulsive wave.

\section{Conclusion}

We have reviewed the Bonnor-Swaninarayan family of boost-rotationally symmetric solutions which was generalised by Bičák, Hoenselaers and Schmidt. We have written these solutions in a unified way paying particular attention to certain limiting cases. These solutions describe the accelerated motion of pairs of particles possibly attached to conical singularities on the axis of symmetry. For the case of multipole particles, we have presented a new simpler form of the solution.

We have specifically investigated the possible null limits of these solutions. In most cases, the limits are trivial or not physically acceptable. However, in some cases we have obtained physically interesting limits which describe snapping or expanding cosmic strings generating spherical impulsive gravitational waves. For multipole particles, we have shown that the internal multipole structure vanishes in this limit, leaving the same solution for a snapping or expanding cosmic string. We have also presented a new solution for a snapping cosmic string of finite length.

\footnotetext{
${ }^{1}$ These statements apply also to the null multipole limits (21) as well as to the null limits of cases $2 \mathrm{a}$ and $3 \mathrm{a}$.
} 


\section{Acknowledgements}

We are grateful to Professor J. Bičák for suggesting that we investigate this topic and for his helpful comments on the draft of the paper. This work was supported by a visiting fellowship from the Royal Society and, in part, by the grant GACR-202/99/0261 of the Czech Republic.

\section{References}

[1] Podolský, J. and Griffiths, J. B. (1999). Class. Quantum Grav. 16, 2937.

[2] Penrose, R. (1972). General Relativity, ed. L. O'Raifeartaigh (Oxford: Clarendon), p 101.

[3] Bičák, J. and Schmidt, B. (1989a). Class. Quantum Grav. 6, 1547.

[4] Bičák, J. and Schmidt, B. (1989b). Phys. Rev. D 40, 1827.

[5] Bičák, J. (1985). In Galaxies, Axisymmetric Systems and Relativity, M.A.H. MacCallum, ed. (Cambridge University Press, Cambridge), p 99.

[6] Bičák, J. (1987). In Gravitation and Geometry, W. Rindler and A. Trautman, eds. (Bibliopolis, Naples), p 55.

[7] Bonnor, W.B. and Swaminarayan, N.S. (1964). Z. Phys. 177, 240.

[8] Bonnor, W.B. (1966). Wissenschaft. Z. der Friedrich-Schiller-Universität Jena 15, 71.

[9] Bonnor, W. B., Griffiths, J. B. and MacCallum, M. A. H. (1994). Gen. Rel. Grav. 26, 687.

[10] Bičák, J. (1968). Proc. Roy. Soc. A 302, 201.

[11] Bičák, J. (1971). In Relativity and Gravitation, C.G. Kuper and A. Peres, eds. (Gordon and Breach, New York), p 47.

[12] Bondi, H. (1957). Rev. Mod. Phys. 29, 423.

[13] Bičák, J., Hoenselaers, C. and Schmidt, B.G. (1983). Proc. Roy. Soc. A 390, 397.

[14] Bičák, J., Hoenselaers, C. and Schmidt, B.G. (1983). Proc. Roy. Soc. A 390, 411.

[15] Gleiser, R. and Pullin, J. (1989). Class. Quantum Grav. 6, L141.

[16] Bičák, J. (1990). Astron. Nachr. 311, 189.

[17] Nutku, Y. and Penrose, R. (1992). Twistor Newsletter No. 34, 9.

[18] Hogan, P. A. (1993). Phys. Rev. Lett. 70, 117.

[19] Podolský, J. and Griffiths, J. B. (2000). Class. Quantum Grav. 17, 1401.

\section{Appendix}

The solution (15) for multipole particles was first presented in [14] using prolate spheroidal coordinates. The expression for $\mu$ and the first term for $\lambda$ in (15) are known from the standard Weyl solutions. However, the second term in $\lambda$ arises as a 'mixed' term representing the interaction between the multipole terms and the 'boost potential'. Denoting the interaction term for the $n^{\text {th }}$ multipole by $\lambda_{n}$, this is required to satisfy the equation

$$
\partial_{\beta} \lambda_{n}=-\frac{2(n+1)}{\sqrt{\beta^{2}-4 \alpha \beta+4}}\left(\frac{P_{n+1}}{\beta^{n+1}}-\frac{2 P_{n}}{\beta^{n+2}}\right),
$$


(this is a correction of equation (3.11) in [14] in which $\frac{1}{2} \lambda_{n}=\gamma_{\mathrm{m}, n}-\beta^{-(n+1)} P_{n}$ ) where the independent coordinates are $\alpha=(1-x y) /(x-y)$ and $\beta=x-y$, and the Legendre polynomials have argument $\alpha$. We have found that this can be integrated directly by introducing the function

$$
\Lambda_{n}=\lambda_{n}-\frac{1}{2} \lambda_{n-1} .
$$

Using the recurrence relation for Legendre polynomials and (37), we find that $\Lambda_{n}$ must satisfy

$$
\partial_{\beta} \Lambda_{n}=-\partial_{\beta}\left(\beta^{-(n+1)} \sqrt{\beta^{2}-4 \alpha \beta+4} P_{n}\right),
$$

which can immediately be integrated. Setting the integration constant to zero and using a recurrence formula derived from (38) with $\lambda_{0}=-\beta^{-1} \sqrt{\beta^{2}-4 \alpha \beta+4}$, we finally obtain the integral

$$
\lambda_{n}=-\frac{1}{2^{n}} \beta^{-1} \sqrt{\beta^{2}-4 \alpha \beta+4} \sum_{l=0}^{n}\left(\frac{2}{\beta}\right)^{l} P_{l} .
$$

In prolate spheroidal coordinates, this is

$$
\lambda_{n}=-\frac{1}{2^{n}}\left(\frac{x+y}{x-y}\right) \sum_{l=0}^{n}\left(\frac{2}{x-y}\right)^{l} P_{l},
$$

which, with the coefficient $M_{n}$, gives the second term in (15) above.

The equivalent expression presented in [14], equation (3.13), is

$$
\lambda_{n}=-\frac{1}{2^{n}}\left(\frac{x+y}{x-y}\right)(n+1)\left(P_{n} f_{n+1}-P_{n+1} f_{n}\right),
$$

where

$$
f_{n+1}=\sum_{k=0}^{n} \frac{1}{k+1} P_{n-k} \sum_{l=0}^{k}\left(\frac{2}{x-y}\right)^{l} P_{k-l} .
$$

Note that in equation (3.13) of [14], there is also a term $P_{n} f_{n+1}(\infty)-P_{n+1} f_{n}(\infty)$, where $f_{n+1}(\infty)=\sum_{k=0}^{n} \frac{1}{k+1} P_{n-k} P_{k}$. However, using the identity $(n+1)\left(P_{n+1} Q_{n}-P_{n} Q_{n+1}\right)=1$ and the standard definition $Q_{n}(\alpha)=\frac{1}{2} P_{n}(\alpha) \ln [(1+\alpha) /(1-\alpha)]-f_{n}(\infty)$, it can easily be shown that $(n+1)\left[P_{n} f_{n+1}(\infty)-P_{n+1} f_{n}(\infty)\right]=1$. This term thus represents only an additive constant which can be omitted.

Since both the solutions (39) and (40) satisfy the same differential equations, they can differ only by a constant. However, by considering the particular value at $x=1, y=0$, it can be seen that this constant must be zero. From this we may now deduce the relation

$$
(n+1)\left(P_{n} f_{n+1}-P_{n+1} f_{n}\right)=\sum_{l=0}^{n}\left(\frac{2}{x-y}\right)^{l} P_{l} .
$$

By expanding the left-hand side in powers of $2 /(x-y)$ and comparing coefficients, we obtain the following non-trivial identity for the Legendre polynomials.

Theorem: For non-negative integers $m$ and $n$, with $m \leq n$, define the functions $X_{n}^{(m)}(\alpha)$ by

$$
X_{n}^{(m)} \equiv \sum_{k=m}^{n} \frac{1}{k+1} P_{n-k} P_{k-m}
$$

where $P_{n}(\alpha)$ are Legendre polynomials, and $X_{m-1}^{(m)}=0$. Then

$$
P_{n} X_{n}^{(m)}-P_{n+1} X_{n-1}^{(m)}=\frac{1}{n+1} P_{m}
$$

for arbitrary $m$ and $n$ and for arbitrary value of the argument $\alpha$.

The left-hand side of the above identity is a polynomial of order $2 n-m$. It can therefore be expanded as a series of Legendre polynomials. However, remarkably, all the coefficients vanish identically except for one. 